\title{
SOURCE-PANC: A Prediction Model for Patients With Metastatic Pancreatic Ductal Adenocarcinoma Based on Nationwide Population-Based Data
}

\author{
Héctor G. van den Boorn, MSc ${ }^{1, *}$; Willemieke P.M. Dijksterhuis, MD 1,2,*; Lydia G.M. van der Geest, PhD2; \\ Judith de Vos-Geelen, MD"; Marc G. Besselink, MD, PhD³; Johanna W. Wilmink, MD, PhD'; \\ Martijn G.H. van Oijen, $\mathrm{PhD}^{1,2}$; and Hanneke W.M. van Laarhoven, MD, PhD, PhD ${ }^{1}$
}

\begin{abstract}
Background: A prediction model for overall survival (OS) in metastatic pancreatic ductal adenocarcinoma (PDAC) including patient and treatment characteristics is currently not available, but it could be valuable for supporting clinicians in patient communication about expectations and prognosis. We aimed to develop a prediction model for OS in metastatic PDAC, called SOURCE-PANC, based on nationwide population-based data. Materials and Methods: Data on patients diagnosed with synchronous metastatic PDAC in 2015 through 2018 were retrieved from the Netherlands Cancer Registry. A multivariate Cox regression model was created to predict OS for various treatment strategies. Available patient, tumor, and treatment characteristics were used to compose the model. Treatment strategies were categorized as systemic treatment (subdivided into FOLFIRINOX, gemcitabine/nab-paclitaxel, and gemcitabine monotherapy), biliary drainage, and best supportive care only. Validation was performed according to a temporal internalexternal cross-validation scheme. The predictive quality was assessed with the $\mathrm{C}$-index and calibration. Results: Data for 4,739 patients were included in the model. Sixteen predictors were included: age, sex, performance status, laboratory values (albumin, bilirubin, CA19-9, lactate dehydrogenase), clinical tumor and nodal stage, tumor sublocation, presence of distant lymph node metastases, liver or peritoneal metastases, number of metastatic sites, and treatment strategy. The model demonstrated a C-index of 0.72 in the internal-external cross-validation and showed good calibration, with the intercept and slope $95 \%$ confidence intervals including the ideal values of 0 and 1 , respectively. Conclusions: A population-based prediction model for OS was developed for patients with metastatic PDAC and showed good performance. The predictors that were included in the model comprised both baseline patient and tumor characteristics and type of treatment. SOURCE-PANC will be incorporated in an electronic decision support tool to support shared decision-making in clinical practice.
\end{abstract}

J Natl Compr Canc Netw 2021;19(9):1045-1053 doi: $10.6004 /$ jnccn.2020.7669

\section{Background}

In sharp contrast to mortality rates for virtually all other malignancies, those for pancreatic ductal adenocarcinoma (PDAC) have not declined since 2000. ${ }^{1}$ Poor 5-year survival ${ }^{2}$ is in part due to the fact that more than half of patients present with metastatic disease, ${ }^{3,4}$ and these patients usually have a prognosis of only a few months. ${ }^{5,6}$ Predicting the exact survival time at diagnosis is challenging because of the heterogeneity of patients and tumors and differences in treatment of metastatic disease. Tools that can accurately predict survival while taking individual characteristics and treatments into account can be helpful for clinicians and patients when making treatment decisions.

Within the past decade, the emergence of prediction models in various cancer types has contributed to assessment of individually aligned prediction of prognosis and support of shared decision-making in clinical practice. $^{7}$ These models based on patient, tumor, and treatment characteristics aim to predict survival for individual patients. In PDAC, these models have been focused mainly on patients with localized disease. ${ }^{8-14}$ The limited number of models that included patients with advanced disease were based on a small number of patients, ${ }^{15}$ developed for specific patient groups (eg, patients after firstline systemic treatment ${ }^{16}$ or treated with gemcitabine-based

${ }^{1}$ Department of Medical Oncology, Cancer Center Amsterdam, Amsterdam UMC, University of Amsterdam, Amsterdam; ${ }^{2}$ Department of Research and Development, Netherlands Comprehensive Cancer Organisation, Utrecht; ${ }^{3}$ Department of Surgery, Cancer Center Amsterdam, Amsterdam UMC, University of Amsterdam, Amsterdam; and ${ }^{4}$ Division of Medical Oncology, Department of Internal Medicine, GROW-School for Oncology and Developmental Biology, Maastricht University Medical Centre, Maastricht, the Netherlands.

*These authors contributed equally to this study.

See JNCCN.org for supplemental online content. 
regimens ${ }^{17-19}$ ), based on patients included in trials only, ${ }^{19,20}$ limited to patients with locally advanced disease, ${ }^{17,21}$ or only described together with patients with localized diasease. ${ }^{22}$ Moreover, they all did not take into account the different palliative systemic treatment options that are currently available for PDAC..$^{23-25}$

Shared decision-making becomes increasingly important in clinical practice. ${ }^{26}$ In patients with PDAC and metastases at initial diagnosis, median overall survival (OS) in the real-world setting (ie, outside of clinical trials) ranges from 2.3 to 5.9 months in patients who receive best supportive care (BSC) only or systemic treatment, respectively. ${ }^{6}$ Given the relatively marginal survival benefits, personalized patient information on treatment outcomes is of paramount importance. Multiple studies have shown that communication about prognosis is a challenge for physicians. ${ }^{27}$ Prediction models can be helpful in supporting communication between physicians and patients regarding expectations and prognosis, and can enhance shared decision-making. ${ }^{7}$

The aim of this study was therefore to create a prediction model, called SOURCE-PANC (stimulating evidence-based, personalized, and tailored information provision to improve decision-making after pancreatic cancer diagnosis). The model was based on a large nationwide cohort using population-based data, with the goal of enabling prediction of OS from diagnosis in patients with metastatic PDAC undergoing palliative systemic treatment, biliary drainage, or BSC only.

\section{Materials and Methods}

\section{Patient Selection}

Data from patients with a confirmed PDAC (C25 according to ICD-O- $3^{28}$ ) or a nonconfirmed supposed adenocarcinoma (see supplemental eTable 1, available with this article at JNCCN.org) and synchronous metastases (T1-4,xN0-2, xM1) diagnosed between 2015 and 2018 were retrieved from the Netherlands Cancer Registry (NCR). The NCR is a population-based registry that includes all cancer diagnoses from the total Dutch population ( $>17$ million inhabitants). Data on patient and tumor characteristics and treatment are extracted from medical records by trained registrars and include information about the patient (age, performance status, other cancer diagnosis, comorbidities), tumor (TNM stage, tumor biology, location of metastases), diagnosis (type of hospital, multidisciplinary consult, exploratory surgery), and treatment (systemic treatment, radiotherapy, palliative interventions, such as stents/drainages/bypasses or BSC only). Data on vital status were obtained through annual linkage to the Dutch Personal Records Database and updated until February 1, 2020.

A total of 5,310 patients with metastatic PDAC were selected from the NCR. Patients aged $<18$ years or diagnosed at autopsy were not included. Patients who died within 14 days after diagnosis were excluded $(n=571)$; a prediction model is not applicable in these cases, because physicians will be able to predict the poor survival themselves in most cases and most probably would not consider starting a treatment other than BSC. ${ }^{29} \mathrm{~A}$ total of 4,739 patients remained for development of the prediction model.

Treatment

Palliative treatment strategies were categorized as follows:

1. Systemic treatment: if patients received systemic therapy $(n=1,429)$

2. Biliary drainage: if patients were not treated with systemic therapy but received a biliary stent or percutaneous biliary drainage $(n=722)$

3. BSC: if patients did not receive systemic treatment or biliary drainage $(n=2,588)$

Subsequently, systemic therapy regimens were subdivided as follows:

1. Fluoropyrimidine, platinum, and irinotecan (eg, FOLFIRINOX [5-FU/leucovorin/oxaliplatin/ irinotecan])

2. Regimens with gemcitabine and nab-paclitaxel

3. Gemcitabine monotherapy

4. Other regimens

Predictor Preselection

Predictors were selected based on availability in the NCR. The predictors based on international consensus identified in the Consensus Statement on Mandatory Measurements in Unresectable Pancreatic Cancer Trials $(\mathrm{COMM}-\mathrm{PACT})^{30}$ served as guidance for the selection. The COMM-PACT predictors include mandatory and recommended baseline and prognostic characteristics that are used in pancreatic cancer studies on systemic treatment to adequately compare outcomes. Depending on availability in the NCR, these factors were used to construct the model, in addition to other potential predictive variables that were available in the NCR. These additional predictive variables were only preselected if they met the following criteria: the number of missing values did not exceed $50 \%$ and the variable was not constant across all patients; that is, the variable could potentially improve the model because it discriminated between patients.

\section{Outcome}

The outcome of the prediction model is OS, which was measured from the date of diagnosis to the date of death or of last follow-up when the patient was censored.

\section{Model Development}

The prediction model development followed the same steps as described previously. ${ }^{31}$ In short, the following analysis was performed. Cox proportional hazards 


\section{Table 1. Baseline Characteristics}

\begin{tabular}{|c|c|}
\hline Characteristic & n (\%) \\
\hline Total, N & 4,739 \\
\hline OS, median (IQR), mo & $2.5(2.4-2.6)$ \\
\hline Age, mean (SD), y & $69.49(10.26)$ \\
\hline BMI, mean (SD), $\mathrm{kg} / \mathrm{m}^{2}$ & $24.71(4.10)$ \\
\hline Missing & $3,322(70.1)$ \\
\hline \multicolumn{2}{|l|}{ Sex } \\
\hline Male & $2,444(51.6)$ \\
\hline Female & $2,295(48.4)$ \\
\hline \multicolumn{2}{|l|}{ WHO performance status } \\
\hline 0 & $735(15.5)$ \\
\hline 1 & $1,077(22.7)$ \\
\hline 2 & 509 (10.7) \\
\hline$\geq 3$ & $356(7.5)$ \\
\hline Missing & $2,062(43.5)$ \\
\hline Albumin, mean (SD), g/L & $36.18(7.84)$ \\
\hline Missing & $1,517(32.0)$ \\
\hline Bilirubin, mean (SD), $\mu \mathrm{mol} / \mathrm{L}$ & $65.81(107.72)$ \\
\hline Missing & 409 (8.6) \\
\hline CRP, mean (SD), mg/L & $52.18(65.37)$ \\
\hline Missing & $906(19.1)$ \\
\hline CA 19-9, mean (SD), kU/L & $3,729.31(3,833.56)$ \\
\hline Missing & $1,806(38.1)$ \\
\hline Creatinine, mean (SD), $\mu \mathrm{mol} / \mathrm{L}$ & $75.93(32.87)$ \\
\hline Missing & $596(12.6)$ \\
\hline Hemoglobin, mean (SD), $\mathrm{mmol} / \mathrm{L}$ & $7.90(1.11)$ \\
\hline Missing & $152(3.2)$ \\
\hline $\mathrm{LDH}$, mean (SD), U/L & $281.97(181.28)$ \\
\hline Missing & $608(12.8)$ \\
\hline \multicolumn{2}{|l|}{ Clinical T stage } \\
\hline 1 & 185 (3.9) \\
\hline 2 & $1,206(25.4)$ \\
\hline 3 & $1,314(27.7)$ \\
\hline 4 & $1,406(29.7)$ \\
\hline Missing & $628(13.3)$ \\
\hline \multicolumn{2}{|l|}{ Clinical $\mathrm{N}$ stage } \\
\hline 0 & $1,979(41.8)$ \\
\hline 1 & $1,657(35.0)$ \\
\hline 2 & $223(4.7)$ \\
\hline Missing & 880 (18.6) \\
\hline
\end{tabular}

(continued)

regression with Efron baseline hazard estimation was used to predict survival. Missing data were multiply imputed $(n=10)$ according to the MICE (multivariate imputation by chained equations) algorithm during the

\begin{tabular}{|c|c|}
\hline Characteristic & n (\%) \\
\hline \multicolumn{2}{|l|}{ Tumor topography } \\
\hline Head of pancreas & $1,931(40.7)$ \\
\hline Body of pancreas & $835(17.6)$ \\
\hline Tail of pancreas & $1,155(24.4)$ \\
\hline Overlapping lesion & $499(10.5)$ \\
\hline Pancreas NOS & $319(6.7)$ \\
\hline \multicolumn{2}{|l|}{ Morphology } \\
\hline Adenocarcinoma & $4,739(100.0)$ \\
\hline \multicolumn{2}{|l|}{ Differentiation grade } \\
\hline 1 & $41(0.9)$ \\
\hline 2 & $217(4.6)$ \\
\hline 3 & $289(6.1)$ \\
\hline Missing & $4,192(88.5)$ \\
\hline \multicolumn{2}{|l|}{ Distant LN metastasis only } \\
\hline No & $4,590(96.8)$ \\
\hline Yes & $151(3.2)$ \\
\hline \multicolumn{2}{|l|}{ Liver metastasis } \\
\hline No & $1,201(25.3)$ \\
\hline Yes & $3,538(74.7)$ \\
\hline \multicolumn{2}{|l|}{ Peritoneal metastasis } \\
\hline No & $3,523(74.3)$ \\
\hline Yes & $1,216(25.7)$ \\
\hline \multicolumn{2}{|l|}{ Pulmonary metastasis } \\
\hline No & $3,778(79.8)$ \\
\hline Yes & $961(20.2)$ \\
\hline Metastatic sites, mean (SD), $n$ & $1.55(0.83)$ \\
\hline \multicolumn{2}{|l|}{ Type of treatment } \\
\hline Best supportive care only & $2,588(54.6)$ \\
\hline Biliary drainage only & $722(15.2)$ \\
\hline FOLFIRINOX & $921(19.4)$ \\
\hline Gemcitabine monotherapy & $324(6.8)$ \\
\hline Gemcitabine + nab-paclitaxel & $120(2.5)$ \\
\hline Other systemic treatment & $64(1.3)$ \\
\hline
\end{tabular}

Abbreviations: BMI, body mass index; CRP, C-reactive protein; FOLFIRINOX 5-FU/leucovorin/oxaliplatin/irinotecan; IQR, interquartile range; LDH, lactate dehydrogenase; LN, lymph node; NOS, not otherwise specified; OS, overall survival.

validation and creation of the final model. ${ }^{32}$ Next, predictors were selected from among the preselected variables using the bidirectional Akaike information criterion method. Predictors selected in most imputations C-index and calibration were determined. The C-index is a measure of discriminatory ability and typically ranges from 0.5 (chance level) to 1 (perfect discrimination). ${ }^{33,34}$ The calibration refers to the concordance between 
Table 2. Possible Prediction Model Predictors

\begin{tabular}{|c|c|c|c|}
\hline Predictors & NCR Availability & Included in COMM-РACT & $\begin{array}{l}\text { Included in } \\
\text { Final Model }\end{array}$ \\
\hline Age & Yes & Yes, mandatory variable & Yes \\
\hline Albumin level & Yes & Yes, mandatory variable & Yes \\
\hline Alkaline phosphatase level & NA & Yes, recommended variable & No \\
\hline Bilirubin level & Yes & Yes, mandatory variable & Yes \\
\hline CA 19-9 level & Yes & Yes, mandatory variable & Yes \\
\hline CEA level & NA & Yes, recommended variable & No \\
\hline Clinical T stage & Yes & Yes, recommended variable & Yes \\
\hline Clinical $N$ stage & Yes & Yes, recommended variable & Yes \\
\hline CRP level & Yes & Yes, mandatory variable & Yes \\
\hline Creatinine level & Yes & No & No \\
\hline Distant LN metastasis only & Yes & No & Yes \\
\hline Hemoglobin level & Yes & No & No \\
\hline LDH level & Yes & Yes, mandatory variable & Yes \\
\hline Liver metastasis & Yes & Yes, mandatory variable & Yes \\
\hline LN metastasis only & Yes & No & No \\
\hline Neutrophil-to-lymphocyte ratio & NA & Yes, mandatory variable & No \\
\hline Number of metastatic sites & Yes, with a maximum of 4 metastatic sites & Yes, mandatory variable & No \\
\hline Number of metastatic sites & Yes & No & Yes \\
\hline Pain at baseline & NA & Yes, mandatory variable & No \\
\hline Performance status & Yes & Yes, mandatory variable & Yes \\
\hline Peritoneal metastasis & Yes & Yes, mandatory variable & Yes \\
\hline Previous DVT or embolus & NA & Yes, recommended variable & No \\
\hline Primary tumor location & Yes & No & Yes \\
\hline Pulmonary metastasis & Yes & Yes, recommended variable & No \\
\hline QoL global functioning ${ }^{a}$ & NA & Yes, recommended variable & No \\
\hline QoL physical functioning ${ }^{a}$ & NA & Yes, recommended variable & No \\
\hline Sex & Yes & Yes, recommended variable & Yes \\
\hline Type of treatment & Yes & No & Yes \\
\hline
\end{tabular}

Abbreviations: COMM-PACT, Consensus Statement on Mandatory Measurements in Unresectable Pancreatic Cancer Trials; CRP, C-reactive protein; DVT, deep venous thrombosis; LDH, lactate dehydrogenase; LN, lymph node; NA, not available; NCR, Netherlands Cancer Registry; QoL, quality of life.

${ }^{a}$ These factors are relative to the EORTC Quality of Life Questionnaire-Core 30 score.

predicted and observed survival and is displayed in a graph. With perfect calibration, the calibration line has an intercept of 0 and a slope of 1 . These methods were used to create the prediction model. To further assess quality, internal-external temporal cross-validation was used, which mimics an external temporal validation. ${ }^{35}$ With this validation, a model is created based on earlier diagnosis years and validated based on the subsequent year. In this case, a model was first created based on patients diagnosed in 2015 and validated based on those diagnosed in 2016. Next, patients from 2015 to 2016 were used to create a model validated based on patients from 2017. Finally, patients diagnosed in the final year, 2018, were used to validate the prediction model based on patients from 2015 to 2017. Analyses were performed using the RStudio environment with $\mathrm{R}$ version 3.6.2 ( $\mathrm{R}$ Foundation for Statistical Computing).

\section{Availability of the Data}

Data supporting the findings of this study are available from the NCR. ${ }^{36}$ Restrictions apply to the availability of these data, which were used under license for this study.

\section{Ethical Statements}

This report was written in accordance with the TRIPOD guidelines. ${ }^{37}$ According to the Central Committee on Research Involving Human Subjects, this type of study does not require approval from an ethics committee in 


\begin{tabular}{|c|c|}
\hline Variable & $\mathrm{HR}(95 \% \mathrm{Cl})$ \\
\hline Age, $y$ & $1.006(1.003-1.009)$ \\
\hline \multicolumn{2}{|l|}{ Sex } \\
\hline Male & Ref \\
\hline Female & $0.893(0.843-0.947)$ \\
\hline \multicolumn{2}{|c|}{ WHO performance status } \\
\hline 0 & Ref \\
\hline 1 & $1.071(0.994-1.155)$ \\
\hline 2 & $1.239(1.137-1.350)$ \\
\hline$\geq 3$ & $1.443(1.314-1.584)$ \\
\hline Albumin, $\mathrm{g} / \mathrm{L}$ & $0.983(0.979-0.987)$ \\
\hline Bilirubin, $\mu \mathrm{mol} / \mathrm{L}$ & $1.000(1.000-1.001)$ \\
\hline $\mathrm{CRP}, \mathrm{mg} / \mathrm{L}$ & $1.003(1.002-1.003)$ \\
\hline CA 19-9, kU/L & $1.000(1.000-1.000)$ \\
\hline $\mathrm{LDH}, \mathrm{U} / \mathrm{L}$ & $1.001(1.000-1.001)$ \\
\hline \multicolumn{2}{|l|}{ Clinical T stage } \\
\hline 1 & Ref \\
\hline 2 & $1.277(1.102-1.481)$ \\
\hline 3 & $1.376(1.186-1.596)$ \\
\hline 4 & $1.314(1.135-1.523)$ \\
\hline \multicolumn{2}{|l|}{ Clinical N stage } \\
\hline 0 & Ref \\
\hline 1 & $1.118(1.053-1.188)$ \\
\hline 2 & $1.277(1.127-1.448)$ \\
\hline \multicolumn{2}{|l|}{ Primary tumor location } \\
\hline Head of pancreas & Ref \\
\hline Body of pancreas & $1.034(0.947-1.129)$ \\
\hline Tail of pancreas & $1.009(0.928-1.097)$ \\
\hline Overlapping lesion & $1.044(0.942-1.157)$ \\
\hline Pancreas NOS & $1.194(1.055-1.351)$ \\
\hline
\end{tabular}

(continued)

the Netherlands. However, the study was approved by the Privacy Review Board of the NCR and the scientific committee of the Dutch Pancreatic Cancer Group (K18.218). ${ }^{38}$

\section{Results}

\section{Patient Characteristics}

Data on 4,739 patients with metastatic PDAC who were eligible for inclusion were used to create the prediction model. Baseline characteristics of these patients are displayed in Table 1 . Of all patients, $48 \%$ were women, and the mean age was 69.5 years. Most of the primary tumors were located in the head of the pancreas $(41 \%)$, followed by the tail $(24 \%)$ or body $(18 \%)$. An overlapping

\begin{tabular}{|c|c|}
\hline Variable & HR $(95 \% \mathrm{Cl})$ \\
\hline \multicolumn{2}{|l|}{ Distant LN metastasis only } \\
\hline No & Ref \\
\hline Yes & $0.695(0.580-0.833)$ \\
\hline \multicolumn{2}{|l|}{ Liver metastasis } \\
\hline No & Ref \\
\hline Yes & $1.398(1.290-1.515)$ \\
\hline \multicolumn{2}{|l|}{ Peritoneal metastasis } \\
\hline No & Ref \\
\hline Yes & $1.219(1.126-1.321)$ \\
\hline Number of metastatic sites & $1.069(1.027-1.112)$ \\
\hline \multicolumn{2}{|l|}{ Type of treatment } \\
\hline Best supportive care only & Ref \\
\hline Biliary drainage only & $0.252(0.230-0.276)$ \\
\hline FOLFIRINOX & $0.694(0.627-0.769)$ \\
\hline Gemcitabine monotherapy & $0.407(0.361-0.458)$ \\
\hline Gemcitabine + nab-paclitaxel & $0.269(0.223-0.325)$ \\
\hline Other systemic treatment & $0.292(0.226-0.378)$ \\
\hline
\end{tabular}

Abbreviations: CRP, C-reactive protein; FOLFIRINOX, 5-FU/leucovorin/ oxaliplatin/irinotecan; $H R$, hazard ratio; $L D H$, lactate dehydrogenase; $L N$, lymph node; NOS, not otherwise specified.

lesions was found in $11 \%$ of the patients, and in $7 \%$ the location was not specified. Three-fourths of the patients had liver metastases (75\%), and $26 \%$ had peritoneal metastases. Most patients (55\%) received BSC only, followed by FOLFIRINOX (19\%), biliary drainage only (15\%), gemcitabine monotherapy (7\%), gemcitabine + nab-paclitaxel $(3 \%)$, or other systemic treatment $(1 \%)$.

\section{Model Parameters}

All possible prognostic variables based on availability in the NCR and variables regarded as mandatory or recommended variables by COMM-PACT are listed in Table 2. Seven COMM-PACT variables were not available in our dataset (ie, neutrophil-to-lymphocyte ratio, pain at baseline, alkaline phosphatase level, CEA level, previous deep venous thrombosis/embolus, and the global and physical functioning quality-of-life [QoL] scales).

A total of 16 predictors were selected in the final model and are presented in Table 3 with their accompanying hazard ratios. Model parameters include patient (age, sex, and performance status), laboratory (albumin, C-reactive protein, CA 19-9, lactate dehydrogenase, bilirubin levels), and tumor characteristics (clinical tumor and nodal stage, primary tumor location, distant lymph node metastasis only, liver metastasis, peritoneal 


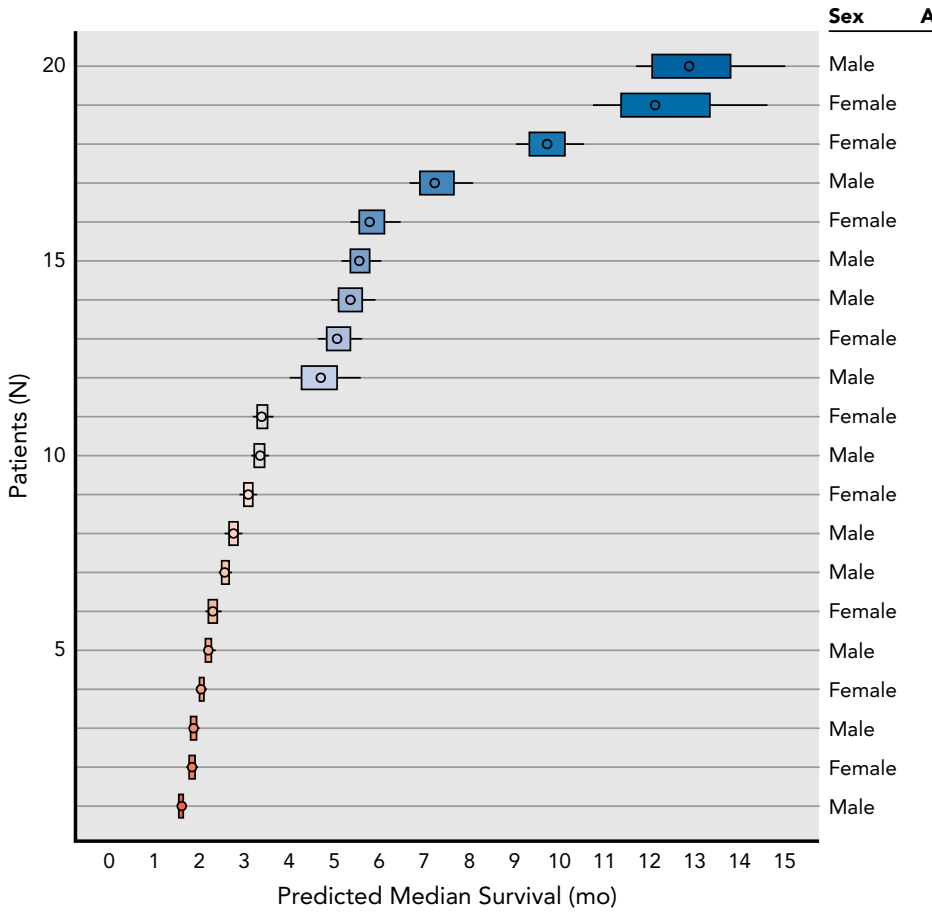

\begin{tabular}{|c|c|c|c|c|}
\hline sex & Age, y & cTNM & Tumor Location & Treatment \\
\hline Male & 52 & cT4N0M1 & Body of pancreas & FOLFIRINOX \\
\hline Female & 75 & cT4N1M1 & Overlapping lesion & Gemcitabine/(Nab-)paclitaxel \\
\hline Eemale & 55 & cT3N1M1 & Head of pancreas & FOLFIRINOX \\
\hline Male & 70 & cT2NOM1 & Overlapping lesion & FOLFIRINOX \\
\hline Female & 46 & cT4N1M1 & Head of Pancreas & FOLFIRINOX \\
\hline Male & 71 & cT2N0M1 & Body of Pancreas & FOLFIRINOX \\
\hline Male & 62 & $\mathrm{cT} 2 \mathrm{~N} 1 \mathrm{M} 1$ & Pancreas NOS & FOLFIRINOX \\
\hline Female & 51 & cT2N0M1 & Pancreas NOS & FOLFIRINOX \\
\hline Male & 79 & cT4N2M1 & Body of Pancreas & Other systemic \\
\hline Female & 67 & cT2N1M1 & Head of Pancreas & Biliary stent \\
\hline Male & 70 & cT2N0M1 & Head of Pancreas & Biliary stent \\
\hline Female & 72 & cT2N0M1 & Head of Pancreas & Biliary stent \\
\hline Male & 64 & cT4N1M1 & Head of Pancreas & Biliary stent \\
\hline Male & 74 & cT2N0M1 & Head of Pancreas & Best supportive care \\
\hline Female & 54 & cT3N0M1 & Head of Pancreas & Biliary stent \\
\hline Male & 60 & cT3N0M1 & Tail of pancreas & Best supportive care \\
\hline Female & 79 & cT2N0M1 & Body of Pancreas & Best supportive care \\
\hline Male & 62 & cT3N0M1 & Tail of Pancreas & Best supportive care \\
\hline Female & 73 & cT4N1M1 & Head of Pancreas & Best supportive care \\
\hline Male & 74 & cT2N0M1 & Body of Pancreas & Best supportive care \\
\hline
\end{tabular}

Figure 1. Predicted and observed median survival. The figure demonstrates the practical applicability of the prediction model in individual patients. The diagram is based on a random sample of 20 patients in the dataset. The bars show the $50 \%$ confidence interval and the lines show the $80 \%$ confidence interval. The table on the right shows the patient characteristics on which the predictions were based.

Abbreviations: FOLFIRINOX, 5-FU/leucovorin/oxaliplatin/irinotecan; NOS, not otherwise specified.

metastasis, number of metastatic sites) and treatment strategy. Compared with BSC only, all treatment strategies (ie, biliary drainage only and systemic treatment strategies) resulted in superior OS. The longest OS was observed in patients who received FOLFIRINOX (hazard ratio, 0.26; 95\% CI, 0.24-0.28) (Table 3). An example of predicted and observed risks in 20 patients is displayed in Figure 1.

\section{Performance}

Model performance statistics are shown in Table 4 . Overall, the model had a good discriminatory ability, with a C-index of 0.72. The model calibration is displayed in Figure 2 and shows an overall good accordance between predicted and observed survival. The calibration is determined at the median OS of 2.5 months after diagnosis. Both the calibration intercept and the slope include the ideal values of 0 and 1 in the $95 \%$ confidence interval, respectively.

\section{Discussion}

This prediction model for patients with synchronous metastatic PDAC is the first model based on a populationbased cohort including a nationwide cohort of patients with metastatic disease diagnosed in 2015 through 2018 $(\mathrm{N}=4,739)$ and various types of (systemic) treatments.
The SOURCE-PANC model stands out with an applicability to a wide range of patients and good internalexternal validation. The model showed good accordance between predicted and observed OS and can be valuable in supporting communication regarding expectations of systemic treatment compared with BSC. The prediction model will be incorporated in a web interface that can be used during consultations in the clinic to contribute to the shared decision-making process. This web interface will be made freely available to the oncologic community and will display personalized survival charts comparing various treatments after input of the model parameters. Results of the exploration of the clinical applicability of an online model for esophagogastric cancer will be used for the implementation of the model for PDAC

\section{Table 4. Model Performance}

\begin{tabular}{|llc|}
\hline & $\begin{array}{c}\text { Complete Model } \\
\text { Value }(\mathbf{9 5 \%} \mathbf{~ C l})\end{array}$ & $\begin{array}{c}\text { Internal- } \\
\text { External Validation } \\
\text { Value }(\mathbf{9 5 \%} \mathbf{~ C I})\end{array}$ \\
\hline C-index & $0.73(0.72-0.74)$ & $0.72(0.71-0.73)$ \\
\hline Calibration intercept & $0.00(0.00-0.00)$ & $0.03(0.00-0.05)$ \\
\hline Calibration slope & $1.00(1.00-1.00)$ & $0.97(0.92-1.01)$ \\
\hline Calibration deviance & $0.00(0.00-0.00)$ & $0.01(0.00-0.02)$ \\
\hline
\end{tabular}




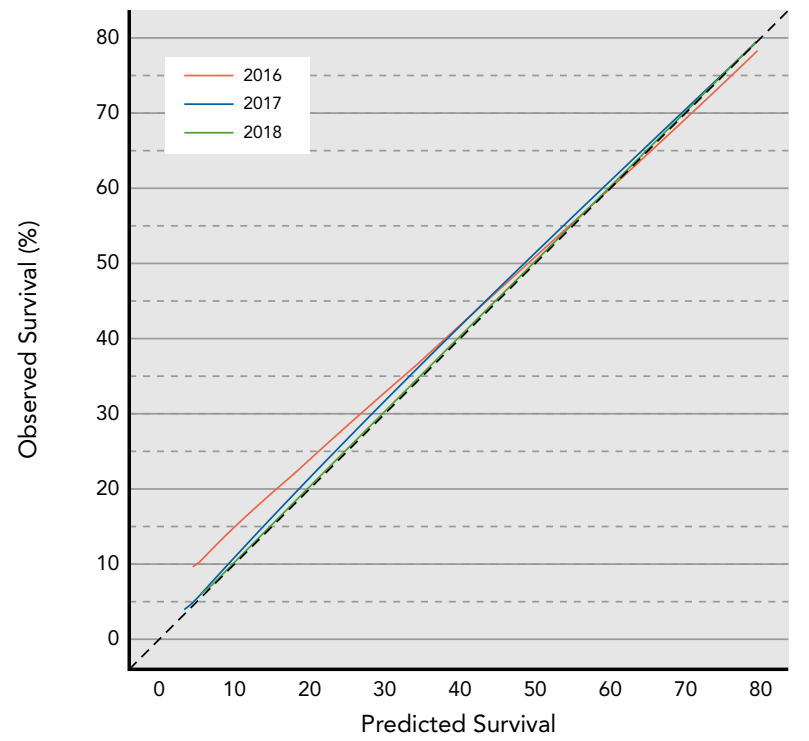

Figure 2. Model calibration. The figure shows the accordance between survival predicted by the model and observed survival for each validation cohort.

(ClinicalTrials.gov identifier: NCT04232735). ${ }^{31}$ Prognostic COMM-PACT variables that were identified by experts in the field of pancreatic cancer were added to the model..$^{30}$ These possible predictors were selected for use as prognostic variables in randomized controlled trials investigating first-line systemic treatment in unresectable or metastatic pancreatic cancer. The variables include common baseline characteristics that are collected routinely in general clinical practice and are therefore easy to add to a model that could be helpful in predicting OS for various treatments while taking into account these prognostic features. However, not all mandatory and recommended predictors that were defined by the COMMPACT study were available in the dataset, and some predictors were missing in a considerable number of patients (eg, performance status, which was missing in $44 \%$ ). This could have impaired the model's performance.

Major strengths of this study are the inclusion of population-based data that represent all patients with metastatic PDAC in clinical practice and the addition of different systemic treatment regimens to the model. We categorized systemic treatment into the most frequently applied regimens (ie, FOLFIRINOX, ${ }^{23}$ gemcitabine + nab-paclitaxel, ${ }^{24}$ gemcitabine monotherapy, ${ }^{25}$ or other treatment regimens) The actual survival benefit of the regimens can be assessed in comparison with each other or with BSC only. As a result, patients should consider the possible benefits of FOLFIRINOX in terms of OS against the increased risk of toxicity of this regimen compared with gemcitabine + nab-paclitaxel or gemcitabine monotherapy. ${ }^{23,39}$

With a validated C-index of 0.72 , the model can be regarded as adequately discriminative, and the model's C-index is larger than that of most other similar models. ${ }^{15-22}$ The model calibration indicates a close coherence between predicted and observed survival. Moreover, validation was performed according to a temporal internal-external scheme resembling a true external validation in future cohorts. External validity of the model with similar cohorts is needed for further verification of the model's accuracy and is crucial to application in clinical practice. ${ }^{40}$ Therefore, the next step will be to validate the model in a different population, such as by using more recent data from the NCR or data from the Belgian Cancer Registry, as has been performed previously. ${ }^{41}$ Use of more recent data from the NCR would be preferable, because differences in registration practices, interpretations, and missing variables in Belgian data may impair the validation. ${ }^{41}$

This study has some limitations. First, we had information available only on the initial treatment and did not know any therapy beyond first-line treatments. Although we expect only a small number of the patients will eventually be eligible to receive these treatments, treatment options beyond first line are expanding. ${ }^{42,43}$ Currently, additional follow-up data are collected in the NCR, and an update of the model that includes secondline systemic treatment strategies will be performed when these data are available. Second, the model focuses only on survival, whereas QoL is also of prime importance to these patients. ${ }^{44}$ Improvement in QoL has been reported for those receiving systemic therapy, ${ }^{45,46}$ biliary stents, ${ }^{47}$ and radiotherapy, ${ }^{48}$ whereas fatigue, pain, and treatment-related toxicity are associated with decreased QoL. ${ }^{44,49}$ We are currently collecting patientreported outcome measures on QoL and will add this information to the model once a sufficient amount of data has been collected. ${ }^{50}$ Third, we did not have data on treatment toxicity, which may be an important factor for patients to consider during treatment decision-making. Furthermore, apart from the number of comorbidities, we did not have data that included comorbidity severity, such as the Charlson comorbidity index. We aim to incorporate these data into the model in the future as well.

\section{Conclusions}

The prediction model developed in this study is the first to present OS outcomes in patients with synchronous metastatic PDAC based on a nationwide cohort. SOURCE-PANC can be used to predict OS with good accordance and calibration. Based on this model, 
a decision support tool will be created to support clinicians in communicating with their patients regarding expectations and prognosis.

\section{Acknowledgments}

The authors thank the registration team of the Netherlands Comprehensive Cancer Organisation (IKNL) for the collection of data for the Netherlands Cancer Registry.

Submitted August 5, 2020; final revision received October 10, 2020 accepted for publication October 12, 2020.

Published online July 21, 2021.

Author contributions: Study design: van den Boorn, Dijksterhuis, van Oijen, van Laarhoven. Data collection: van den Boorn, Dijksterhuis, van der Geest. Data analysis: van den Boorn, Dijksterhuis. Manuscript preparation: van den Boorn, Dijksterhuis, van Oijen, van Laarhoven.
Disclosures: Dr de Vos-Geelen has reported receiving nonfinancial support from BTG Specialty Pharmaceuticals and Servier; serving as a consultant for Amgen, AstraZeneca, Merck Sharp \& Dohme, Pierre Fabre, Servier, and Shire; and receiving grant/research support from Servier. Dr. Wilmink has reported serving as a consultant for Celgene, Merck, and Servier; and receiving unrestricted research funding from Bayer, Celgene, Merck Serono, Nordic Bioscience, and Servier. Dr. van Oijen has reported receiving grant/research support from Bayer, Bristol Myers Squibb, Lilly, Merck Serono, Nordic Pharma and Roche. Dr. van Laarhoven has reported serving as a consultant for Bristol Myers Squibb, Celgene, Lilly/lmClone, Merck Sharp \& Dohme, Nordic Pharma, and Servier; and receiving grant/research support from Bayer Schering Pharma, Bristol Myers Squibb, Celgene, Lilly, Merck Serono, Merck Sharp \& Dohme, Nordic Pharma, Philips Healthcare, Roche, and Servier. The remaining authors have reported that they have not received any financial consideration from any person or organization to support the preparation, analysis, results, or discussion of this article.

Correspondence: Hanneke W.M. van Laarhoven, MD, PhD, PhD, Departmen of Medical Oncology, Cancer Center Amsterdam, Amsterdam UMC, University of Amsterdam, Meibergdreef 9, 1105 AZ Amsterdam, the Netherlands. Email: h.vanlaarhoven@amsterdamumc.nl

\section{References}

1. Bertuccio $P$, Alicandro $G$, Malvezzi $M$, et al. Cancer mortality in Europe in 2015 and an overview of trends since 1990. Ann Oncol 2019:30: 1356-1369.

2. Lepage $C$, Capocaccia R, Hackl M, et al. Survival in patients with primary liver cancer, gallbladder and extrahepatic biliary tract cancer and pancreatic cancer in Europe 1999-2007: results of EUROCARE-5. Eur J Cancer 2015;51:2169-2178.

3. Baxter NN, Whitson BA, Tuttle TM. Trends in the treatment and outcome of pancreatic cancer in the United States. Ann Surg Oncol 2007;14: $1320-1326$

4. van der Geest LGM, van Eijck CHJ, Groot Koerkamp B, et al. Trends in treatment and survival of patients with nonresected, nonmetastatic pancreatic cancer: a population-based study. Cancer Med 2018;7: 4943-4951.

5. van der Geest LGM, Haj Mohammad N, Besselink MGH, et al. Nationwide trends in chemotherapy use and survival of elderly patients with metastatic pancreatic cancer. Cancer Med 2017;6:2840-2849.

6. Latenstein AEJ, van der Geest LGM, Bonsing BA, et al. Nationwide trends in incidence, treatment and survival of pancreatic ductal adenocarcinoma. Eur J Cancer 2020;125:83-93.

7. Vickers AJ. Prediction models in cancer care. CA Cancer J Clin 2011;61: 315-326.

8. de Castro SM, Biere SSAY, Lagarde SM, et al. Validation of a nomogram for predicting survival after resection for adenocarcinoma of the pancreas. $\mathrm{Br}$ J Surg 2009;96:417-423.

9. Smith BJ, Mezhir JJ. An interactive Bayesian model for prediction of lymph node ratio and survival in pancreatic cancer patients. J Am Med Inform Assoc 2014;21:e203-211.

10. Haga $Y$, Wada $Y$, Saitoh $T$, et al. Value of general surgical risk models for predicting postoperative morbidity and mortality in pancreatic resections for pancreatobiliary carcinomas. J Hepatobiliary Pancreat Sci 2014;21: 599-606.

11. Lee $\mathrm{KH}$, Lee JK, Choi DW, et al. Postoperative prognosis prediction of pancreatic cancer with seven microRNAs. Pancreas 2015;44:764-768.

12. He C, Mao $Y$, Wang J, et al. Nomograms predict long-term survival for patients with periampullary adenocarcinoma after pancreatoduodenectomy. BMC Cancer 2018;18:327.

13. Huang $L$, Balavarca $Y$, van der Geest $L$, et al. Development and validation of a prognostic model to predict the prognosis of patients who underwent chemotherapy and resection of pancreatic adenocarcinoma: a large international population-based cohort study. BMC Med 2019;17:66

14. Latenstein AEJ, van Roessel S, van der Geest LGM, et al. Conditional survival after resection for pancreatic cancer: a population-based study and prediction model. Ann Surg Oncol 2020;27:2516-2524.

15. Forssell H, Wester $M$, Akesson $\mathrm{K}$, et al. A proposed model for prediction of survival based on a follow-up study in unresectable pancreatic cancer. BMJ Open 2013;3:e004064.

16. Vienot $A$, Beinse $G$, Louvet $C$, et al. Overall survival prediction and usefulness of second-line chemotherapy in advanced pancreatic adenocarcinoma. J Natl Cancer Inst 2017:109:djx037.
17. Hamada $T$, Nakai $Y$, Yasunaga $H$, et al. Prognostic nomogram for nonresectable pancreatic cancer treated with gemcitabine-based chemotherapy. Br J Cancer 2014;110:1943-1949.

18. Deng $\mathrm{QL}$, Dong $\mathrm{S}$, Wang $\mathrm{L}$, et al. Development and validation of a nomogram for predicting survival in patients with advanced pancreatic ductal adenocarcinoma. Sci Rep 2017;7:11524.

19. Goldstein D, Von Hoff DD, Chiorean EG, et al. Nomogram for estimating overall survival in patients with metastatic pancreatic cancer. Pancreas 2020:49:744-750

20. Hang J, Wu L, Zhu L, et al. Prediction of overall survival for metastatic pancreatic cancer: development and validation of a prognostic nomogram with data from open clinical trial and real-world study. Cancer Med 2018;7:2974-2984.

21. Vernerey D, Huguet F, Vienot A, et al. Prognostic nomogram and score to predict overall survival in locally advanced untreated pancreatic cancer (PROLAP). Br J Cancer 2016;115:281-289.

22. Song W, Miao DL, Chen L. Nomogram for predicting survival in patients with pancreatic cancer. Onco Targets Ther 2018;11:539-545

23. Conroy $T$, Desseigne F, Ychou M, et al. FOLFIRINOX versus gemcitabine for metastatic pancreatic cancer. N Engl J Med 2011;364: 1817-1825.

24. Von Hoff DD, Ervin T, Arena FP, et al. Increased survival in pancreatic cancer with nab-paclitaxel plus gemcitabine. N Engl J Med 2013;369: 1691-1703.

25. Burris HA III, Moore MJ, Andersen J, et al. Improvements in survival and clinical benefit with gemcitabine as first-line therapy for patients with advanced pancreas cancer: a randomized trial. J Clin Oncol 1997;15: 2403-2413.

26. Stiggelbout AM, Van der Weijden T, De Wit MPT, et al. Shared decision making: really putting patients at the centre of healthcare. BMJ 2012;344 e256.

27. van de Water LF, van Kleef JJ, Dijksterhuis WPM, et al. Communicating treatment risks and benefits to cancer patients: a systematic review of communication methods. Qual Life Res 2020;29:1747-1766.

28. Fritz A, Percy C, Jack A, et al. International Classification of Diseases for Oncology, 3rd ed. Geneva, Switzerland: World Health Organization; 2000.

29. Palliatieve Sedatie. Accessed February 13, 2021. Available at: https:// www.pallialine.nl/sedatie

30. ter Veer E, van Rijssen LB, Besselink MG, et al. Consensus statement on mandatory measurements in pancreatic cancer trials (COMM-PACT) for systemic treatment of unresectable disease. Lancet Oncol 2018;19: e151-160.

31. van den Boorn HG, Abu-Hanna A, Ter Veer E, et al. SOURCE: a registrybased prediction model for overall survival in patients with metastatic oesophageal or gastric cancer. Cancers (Basel) 2019;11:187

32. van Buuren S, Groothuis-Oudshoorn K. MICE: Multivariate Imputation by Chained Equations in R. J Stat Softw 2011;45:67. 
33. Steyerberg EW, Vickers AJ, Cook NR, et al. Assessing the performance of prediction models: a framework for traditional and novel measures. Epidemiology 2010;21:128-138.

34. Gerds TA, Schumacher M. Consistent estimation of the expected Brier score in general survival models with right-censored event times. Biom J 2006;48:1029-1040.

35. Steyerberg EW, Harrell FE Jr. Prediction models need appropriate internal, internal-external, and external validation. J Clin Epidemiol 2016; 69:245-247.

36. DUCG. Dutch Upper GI Cancer Group. DUCG 2015. Available at: www. ducg.nl

37. Collins GS, Reitsma JB, Altman DG, et al. Transparent reporting of a multivariable prediction model for individual prognosis or diagnosis (TRIPOD): the TRIPOD statement. BMJ 2015;350:g7594

38. Strijker M, Mackay TM, Bonsing BA, et al. Establishing and coordinating a nationwide multidisciplinary study group: lessons learned by the Dutch Pancreatic Cancer Group. Ann Surg 2020;271:e102-104.

39. Tahara J, Shimizu K, Otsuka N, et al. Gemcitabine plus nab-paclitaxel vs. FOLFIRINOX for patients with advanced pancreatic cancer. Cancer Chemother Pharmacol 2018;82:245-250.

40. Collins GS, de Groot JA, Dutton S, et al. External validation of multivariable prediction models: a systematic review of methodological conduct and reporting. BMC Med Res Methodol 2014;14:40.

41. van Kleef JJ, van den Boorn HG, Verhoeven RHA, et al. External validation of the Dutch SOURCE survival prediction model in Belgian metastatic oesophageal and gastric cancer patients. Cancers (Basel) 2020;12:834.

42. Wang-Gillam A, Li CP, Bodoky G, et al. Nanoliposomal irinotecan with fluorouracil and folinic acid in metastatic pancreatic cancer after previous gemcitabine-based therapy (NAPOLI-1): a global, randomised, openlabel, phase 3 trial. Lancet 2016;387:545-557.

43. Oettle H, Riess $H$, Stieler JM, et al. Second-line oxaliplatin, folinic acid, and fluorouracil versus folinic acid and fluorouracil alone for gemcitabine-refractory pancreatic cancer: outcomes from the CONKO-003 trial. J Clin Oncol 2014;32:2423-2429.

44. Lewis AR, Pihlak R, McNamara MG. The importance of quality-of-life management in patients with advanced pancreatic ductal adenocarcinoma. Curr Probl Cancer 2018;42:26-39.

45. Glimelius B, Hoffman K, Sjödén PO, et al. Chemotherapy improves survival and quality of life in advanced pancreatic and biliary cancer. Ann Oncol 1996;7:593-600.

46. Gourgou-Bourgade S, Bascoul-Mollevi C, Desseigne F, et al. Impact of FOLFIRINOX compared with gemcitabine on quality of life in patients with metastatic pancreatic cancer: results from the PRODIGE 4/ACCORD 11 randomized trial. J Clin Oncol 2013;31:23-29.

47. Walter D, van Boeckel PGA, Groenen MJM, et al. Higher quality of life after metal stent placement compared with plastic stent placement for malignant extrahepatic bile duct obstruction: a randomized controlled trial. Eur J Gastroenterol Hepatol 2017;29:231-237.

48. Tian Q, Zhang F, Wang Y. Clinical assessment of palliative radiotherapy for pancreatic cancer. Cancer Radiother 2018;22:778-783.

49. Carrato A, Falcone A, Ducreux M, et al. A systematic review of the burden of pancreatic cancer in Europe: real-world impact on survival, quality of life and costs. J Gastrointest Cancer 2015;46:201-211.

50. Coebergh van den Braak RRJ, van Rijssen LB, van Kleef JJ, et al. Nationwide comprehensive gastro-intestinal cancer cohorts: the $3 \mathrm{P}$ initiative. Acta Oncol 2018;57:195-202. 
Supplemental online content for:

\section{SOURCE-PANC: A Prediction Model for Patients With Metastatic Pancreatic Ductal Adenocarcinoma Based on Nationwide Population-Based Data}

Héctor G. van den Boorn, MSc; Willemieke P.M. Dijksterhuis, MD; Lydia G.M. van der Geest, PhD; Judith de Vos-Geelen, MD; Marc G. Besselink, MD, PhD; Johanna W. Wilmink, MD, PhD; Martijn G.H. van Oijen, PhD; and Hanneke W.M. van Laarhoven, MD, PhD, PhD

J Nat/ Compr Canc Netw 2021;19(9):1045-1053

eTable 1: Included ICD-O Morphology Codes 


\section{eTable 1. Included ICD-O Morphology Codes}

$8000,8001,8010,8011,8012,8020,8021,8022,8031,8032,8033,8035,8046,8070,8071,8072,8140,8141,8143,8144,8145,8154,8163,8201,8211,8255$, $8260,8263,8310,8440,8480,8481,8490,8500,8510,8521,8523,8560,8570,8572,8575,8576$ 